\title{
The effect of pupil size and peripheral brightness on detection and discrimination performance
}

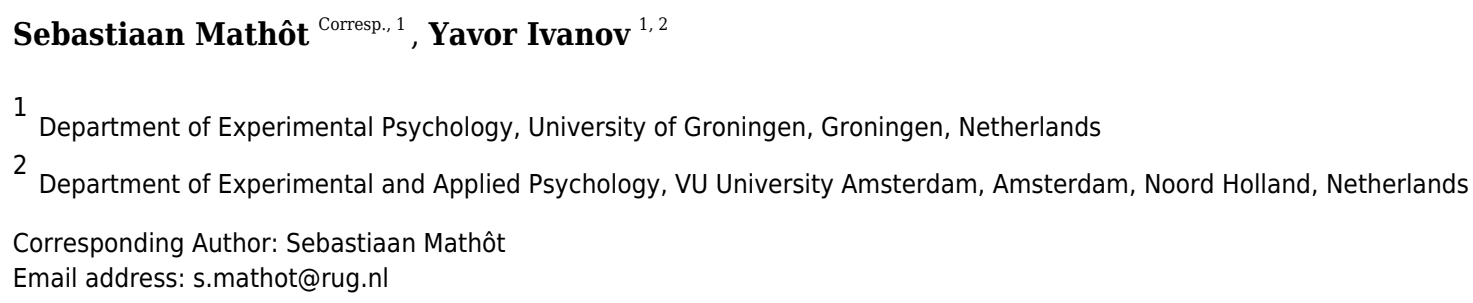

It is easier to read dark text on a bright background (positive polarity) than to read bright text on a dark background (negative polarity). This positive-polarity advantage is often linked to pupil size: A bright background induces small pupils, which in turn increases visual acuity. Here we report that pupil size, when manipulated through peripheral brightness, has qualitatively different effects on discrimination of fine stimuli in central vision and detection of faint stimuli in peripheral vision. Small pupils are associated with improved discrimination performance, consistent with the positive-polarity advantage, but only for very small stimuli that are at the threshold of visual acuity. In contrast, large pupils are associated with improved detection performance. These results are likely due to two pupil-size related factors: Small pupils increase visual acuity, which improves discrimination of fine stimuli; and large pupils increase light influx, which improves detection of faint stimuli. Light scatter is likely also a contributing factor: When a display is bright, light scatter creates a diffuse veil of retinal illumination that reduces perceived image contrast, thus impairing detection performance. We further found that pupil size was larger during the detection task than during the discrimination task, even though both tasks were equally difficult and similar in visual input; this suggests that the pupil may automatically assume an optimal size for the current task. Our results may explain why pupils dilate in response to arousal: This may reflect an increased emphasis on detection of unpredictable danger, which is crucially important in many situations that are characterized by high levels of arousal. Finally, we discuss the implications of our results for the ergonomics of display design. 
1

2

3

4

5

6

7

8

9

10

11

12

13

14

15

16

17

18

19

20

21

22

23 Address for correspondence:

24 Sebastiaan Mathôt

25 University of Groningen

26 Grote Kruisstraat 2/1

27 9712TS Groningen

28 The Netherlands

29 s.mathot@rug.nl

30 https://www.cogsci.nl/smathot
Sebastiaan Mathôt [1,2] and Yavor Ivanov [1,2,3]

1 Department of Psychology, University of Groningen, The Netherlands

2 Research School of Behavioral and Cognitive Neurosciences, University of Groningen, The Netherlands

3 Department of Experimental and Applied Psychology, VU University Amsterdam, The Netherlands 
32 It is easier to read dark text on a bright background (positive polarity) than to read bright text on a dark 33 background (negative polarity). This positive-polarity advantage is often linked to pupil size: A bright 34 background induces small pupils, which in turn increases visual acuity. Here we report that pupil size, when 35 manipulated through peripheral brightness, has qualitatively different effects on discrimination of fine stimuli 36 in central vision and detection of faint stimuli in peripheral vision. Small pupils are associated with improved 37 discrimination performance, consistent with the positive-polarity advantage, but only for very small stimuli 38 that are at the threshold of visual acuity. In contrast, large pupils are associated with improved detection 39 performance. These results are likely due to two pupil-size related factors: Small pupils increase visual acuity, 40 which improves discrimination of fine stimuli; and large pupils increase light influx, which improves detection 41 of faint stimuli. Light scatter is likely also a contributing factor: When a display is bright, light scatter creates a 42 diffuse veil of retinal illumination that reduces perceived image contrast, thus impairing detection 43 performance. We further found that pupil size was larger during the detection task than during the 44 discrimination task, even though both tasks were equally difficult and similar in visual input; this suggests that 45 the pupil may automatically assume an optimal size for the current task. Our results may explain why pupils 46 dilate in response to arousal: This may reflect an increased emphasis on detection of unpredictable danger, 47 which is crucially important in many situations that are characterized by high levels of arousal. Finally, we 48 discuss the implications of our results for the ergonomics of display design.

Keywords: pupillometry, pupil size, pupil light response, display polarity, display design, ergonomics, 50 psychophysics 


\section{The Effect of Pupil Size and Peripheral Brightness on Detection and Discrimination Performance}

You are probably reading this text as dark letters on a bright background. And if not, then you might consider doing so, because it is easier to read dark letters on a bright background (positive polarity) than it is to read bright letters on a dark background (negative polarity). This positive-polarity advantage has been wellestablished in human-factors research (Buchner, Mayr, \& Brandt, 2009; Dobres, Chahine, \& Reimer, 2017; Piepenbrock, Mayr, \& Buchner, 2014b, 2014a; Taptagaporn \& Saito, 1990), and is often studied using proofreading experiments. For example, Piepenbrock et al. (2014b) asked participants to verbally report all misspelled words in a short text. The authors found that participants read faster, and spotted more mistakes, when the text was presented in a positive polarity, as compared to a negative polarity. Findings such as these are among the reasons that most websites and word-processing software use a positive polarity.

Several researchers have suggested that the positive-polarity advantage is linked to pupil size (e.g. Piepenbrock et al., 2014b). When the background of a display is bright, the pupil constricts, compared to when the background is dark; this is the pupil light response (reviewed in Mathôt, 2018; Mathôt \& Van der Stigchel, 2015). In terms of visual perception, there are three main consequences of a bright background and the resulting pupil constriction. The first consequence is negative: A bright background, as any source of brightness, results in light scatter; that is, some of the incoming light is not focused, but instead spreads over a large part of the retina. This results in a diffuse veil of light that reduces perceived image contrast. The second consequence is also negative: Small pupils reduce the amount of light that falls on the retina, and thus reduce the signal-to-noise ratio of the image. The third consequence is positive: Small pupils suffer less from optical distortions that reduce image quality, and thus increase visual acuity (Campbell \& Gregory, 1960; Liang \& Williams, 1997; M. Lombardo \& Lombardo, 2010; Woodhouse, 1975); that is, small pupils result in sharper vision. (Background luminance also affects other aspects of vision, such as contrast sensitivity, in complex ways, as reviewed by Kalloniatis and Luu (1995). Whether, and if so how, these factors also play a role in the positive-polarity advantage is not yet fully clear.)

When reading text that is presented with sufficiently high contrast, as is typically the case in daily life, the advantage of increased visual acuity seems to outweigh the disadvantages of reduced signal-to-noise ratio and increased light scatter. Therefore, it is easier to read dark text on a bright background (when pupils are small), especially when the text is written in a small font (Piepenbrock et al., 2014a). 
81 There is also some neurophysiological evidence that small pupils increase visual acuity. For example,

82 Bombeke and colleagues (2016) manipulated pupil size by having participants covertly attend to either a bright

83 or a dark disk, which respectively constricts or dilates the pupils, without changing eye position or visual input

84 (cf. Binda, Pereverzeva, \& Murray, 2013; Mathôt, van der Linden, Grainger, \& Vitu, 2013). They then briefly

85 presented a task-irrelevant but salient line-grating stimulus in peripheral vision, and measured the $\mathrm{C} 1$, an

86 event-related potential (ERP) component that is associated with activity in primary visual cortex. The

87 amplitude of the $\mathrm{C} 1$ was larger when participants covertly attended the bright disk (resulting in small pupils),

88 compared to when they covertly attended the dark disk (resulting in large pupils). According to the authors,

89 this result was due to the fact that small pupils improved the resolution of the C1-eliciting stimulus, in turn

90 leading to a stronger neural response in primary visual cortex.

91 However, behavioral evidence for a link between pupil size and visual acuity is mixed. In a recent study by 92 Ajasse, Benosman, and Lorenceau (2018), participants made a sequence of eye movements toward a 93 configuration of disks; each disk had a different brightness, and the size of the pupil was therefore different 94 depending on which disk the participant was fixating. While participants were fixating a disk, two gabor 95 patches were briefly and sequentially presented in their visual periphery. The spatial frequency of the gabor 96 patches differed, and participants indicated which of the two had the highest spatial frequency. The authors 97 predicted that performance on this task should increase with decreasing pupil size (and thus with increasing 98 brightness of the fixated disk). However, they found no such relationship; that is, performance did not depend 99 on pupil size.

100 The results of Ajasse and colleagues (2018) show that small pupils do not lead to improved discrimination performance in every situation. Specifically, in their experiment, stimuli were presented in peripheral vision, where acuity is mostly limited by the reduced density of cone photoreceptors; therefore, in peripheral vision, optical blur due to large pupils likely has at most a very small effect on stimulus discrimination. However, the results of Bombeke and colleagues (2016), who also used a peripherally presented stimulus, suggest that under specific conditions a small-pupil advantage can also be found in peripheral vision.

106 In yet other situations, small pupils may even impair visual performance (reviewed in Mathôt, 2018; Mathôt \&

107 Van der Stigchel, 2015). Specifically, detecting faint stimuli in peripheral vision requires a high signal-to-noise 108 ratio of the stimulus relative to the background, and visual acuity is only of secondary importance. In this case, 109 large pupils may improve the signal-to-noise ratio of vision by increasing overall light influx; more 110 specifically, when your aim is to detect a target stimulus against a background, then large pupils will enhance 111 the signal strength of both the target and the background, thereby reducing the influence of noise, in turn 112 making the target more easily distinguishable from the background. Therefore, stimulus detection in the visual 113 periphery should benefit from large pupils. When large pupils are associated with a dark environment, as is 
114 typically the case in real life, this benefit should be even stronger, because the increased signal-to-noise ratio 115 due to large pupils is accompanied by reduced light scatter due to the dark environment. (A subtle, additional 116 complexity comes from the fact that pupil size and light scatter are likely interdependent such that large pupils 117 increase light scatter by increasing light influx. This may be another reason why large pupils are most 118 advantageous in a dark environment, in which light scatter plays a relatively minor role.)

119 However, a study by Thigpen, Bradley, and Keil (2018) suggests that large pupils may not necessarily 'boost' 120 neural responses to visual input. In their study, they presented a rapidly flickering stimulus, and measured so121 called Steady-State Visually Evoked Potentials (ssVEPs): neural oscillations in visual cortex with the same 122 frequency as the inducing stimulus. ssVEP power is believed to reflect the level of neural activity. Crucially, 123 the authors found no relationship between ssVEP power and pupil size, and they interpreted this result as 124 evidence for divisive normalization (Carandini \& Heeger, 2012); that is, they suggested that visual responses, 125 even in early visual cortex, are invariant to overall light influx and thus unaffected by pupil size.

126 Taken together, previous research has provided compelling evidence for an advantage of small pupils (and a 127 bright background) for text reading (Buchner et al., 2009; Dobres et al., 2017; Piepenbrock et al., 2014b, 128 2014a; Taptagaporn \& Saito, 1990). There is also some neurophysiological evidence for a small-pupil benefit 129 for visual acuity (Bombeke et al., 2016; but see Ajasse et al., 2018). In contrast, there is no evidence for a 130 large-pupil advantage for stimulus detection (e.g. Thigpen et al., 2018). Nevertheless, a large-pupil advantage 131 for detection is clearly predicted based on the optical properties of the eye (see Mathôt, 2018; Mathôt \& Van 132 der Stigchel, 2015).

133 The aim of the current study is to demonstrate both a small-pupil advantage for discrimination of stimuli in 134 central vision, and a large-pupil advantage for detection of stimuli in peripheral vision. We will manipulate 135 pupil size by manipulating the brightness of the visual periphery (which likely also affects performance 136 independently of pupil size, as discussed above), while presenting all task-relevant stimuli on a central gray 137 disk of constant brightness.

139 The goal of Experiments 1 and 2 was to investigate whether pupil size, when manipulated through peripheral 140 brightness, differentially affects performance on detection and discrimination tasks. In Experiment 1, 141 participants detected, or discriminated the orientation of, a tilted Gabor patch. In Experiment 2, participants 142 detected, or discriminated the lexicality of, a single word. 


\section{Experiment 1}

145 Participants, Ethics, and Apparatus Nine naive observers participated in the experiment, after providing 146 written informed consent. The experiment was approved by the local ethics committee of Groningen 147 University (16163-SP-NE and 16349-S-NE). Observers were recruited from the community of Groningen 148 University, but for privacy reasons we did not collect specific demographic information. Pupil size was 149 recorded with an EyeLink 1000 (SR Research). Stimuli were presented with OpenSesame 3.1 (Mathôt, Schreij, 150 \& Theeuwes, 2012) on a 27" flat screen Iiyama monitor with a resolution of $1920 \times 1080 \mathrm{px}$. The monitor 151 was not gamma calibrated. Participants were seated with their head on a chin rest about $50 \mathrm{~cm}$ away from the 152 monitor.

153 Pupil-size manipulation Pupil size was manipulated by varying the brightness of the visual periphery (low: $1540.16 \mathrm{~cd} / \mathrm{m}^{2}$, medium: $8.30 \mathrm{~cd} / \mathrm{m}^{2}$, high: $52.26 \mathrm{~cd} / \mathrm{m}^{2}$; see Figure 1), which corresponded to the full display $155\left(49.22^{\circ} \times 27.70^{\circ}\right)$ except for a central gray disk. All task-relevant stimuli were presented on the central gray 156 disk (2.84 cd/ $/ \mathrm{m}^{2}$; diameter: $\left.25.65^{\circ}\right)$ that was kept constant throughout the experiment.

157 Design The experimental task (discrimination or detection) was varied between sessions. One experimental 158 session consisted of five blocks.

159 The first two blocks of each session served to calibrate a Quest adaptive procedure, which varied the properties 160 of the target stimulus (see below) such that accuracy was kept at $75 \%$. During these calibration blocks, 161 peripheral brightness was set to $2.84 \mathrm{~cd} / \mathrm{m}^{2}$. After these two blocks, the Quest procedure was stopped, and the 162 target was kept constant throughout the remainder of the session. Next, participants performed three blocks of 16350 trials. Peripheral brightness was varied between blocks (Figure 1).

164 Block order was fully counterbalanced, such that each possible order occurred once for each participant and 165 task. Half the participants started on the first day with a discrimination session followed by a detection session, 166 vice versa on the second day, etc. The other half of the participants started with a detection session on the first 167 day. In some cases, participants did more than two sessions per day. In total, participants performed 3,000 168 trials across 12 sessions in approximately six hours.

169 Discrimination Task In the discrimination task (see Figure 2a), each trial started with a central fixation dot (a 170 uniform patch with a gaussian envelope with a standard deviation of $0.51^{\circ}[20 \mathrm{px}]$ and a peak brightness of $1714.41 \mathrm{~cd} / \mathrm{m}^{2}$ ) that was removed after $500 \mathrm{~ms}$. After a random interval between 500 and 1,500 ms, drawn from a 172 uniform distribution, a central target stimulus linearly faded in and out over a period of $650 \mathrm{~ms}$. The target was 
173 a centrally presented sinusoidal grating with a gaussian envelope (a Gabor patch) with a standard deviation of

$1740.51^{\circ}(20 \mathrm{px})$. To maintain accuracy at $75 \%$, the spatial frequency $\left(\right.$ range $=[7.8,11.7]$ cycles $\left./{ }^{\circ}\right)$, contrast

$175($ range $=[1,25] \%)$, and orientation $\left(\right.$ range $\left.=[1,2.5]^{\circ}\right)$ of the target was varied with a Quest adaptive procedure

176 during calibration blocks as described above.

177 At any point during the trial, participants pressed the left arrow key if the target was tilted counterclockwise 178 from a vertical orientation, and the right arrow key if it was tilted clockwise (i.e., a two-alternative forced 179 choice). The trial ended $3 \mathrm{~s}$ after the onset of the target. If the participant did not press any key within $3 \mathrm{~s}$, a 180 timeout was registered, and the response was considered incorrect.

181 Detection Task In the detection task (see Figure 2b), each trial started with a central fixation $\operatorname{dot}\left(4.41 \mathrm{~cd} / \mathrm{m}^{2}\right)$ 182 that remained visible throughout the trial. On 50\% of trials, after a random period drawn from a flat 183 distribution between 1 and $2 \mathrm{~s}$, a target stimulus was linearly faded in and out over a period of $650 \mathrm{~ms}$. The 184 target was identical to that of the discrimination task, except that its standard deviation was $1.02^{\circ}(40 \mathrm{px}$; i.e. 185 twice as big), that the spatial frequency was twice as low (range $=[3.9,5.9]$ cycles $\left./{ }^{\circ}\right)$, and that it was presented 186 at a random point on an imaginary circle around the fixation dot with a radius of $7.70^{\circ}(300 \mathrm{px})$.

187 At any point during the trial, participants pressed the space bar when they detected a target, and did not press 188 any key when they did not detect a target. The trial ended $3 \mathrm{~s}$ after the onset of a target (when present), or after 189 a random interval between 4 and $5 \mathrm{~s}$, drawn from a uniform distribution.

\section{Experiment 2}

191 Experiment 2 was in most ways identical to Experiment 1, and only the differences are described below.

192 Participants, Ethics, and Apparatus Nine naive observers, most of whom had not participated in Experiment

193 1, participated in the experiment after providing written informed consent. All participants were native Dutch 194 speakers.

195 Stimulus selection We selected the 750 most highly frequent words between four and six characters from the 196 Dutch Lexicon Project (Keuleers, Diependaele, \& Brysbaert, 2010), after manually (and based on our 197 subjective impression) excluding overly offensive words. For each word, a matching pseudoword was 198 generated with Wuggy (Keuleers \& Brysbaert, 2010).

199 Design Participants performed 1,500 trials across six sessions in approximately three hours. All participants 200 saw all words and pseudowords once in a random order. 
201 Task Targets were (pseudo)words presented in a monospace font (Droid Sans Mono). In the discrimination

202 task, targets were centrally presented, and participants pressed the left arrow key if the target was a 203 pseudoword and the right arrow key if it was a word (i.e. a lexical-decision task). In the detection task, targets 204 were peripherally presented on $50 \%$ of trials, and participants pressed the space bar if they detected a target, 205 and did not press any key otherwise. To maintain accuracy at $75 \%$, the font size and contrast of the target was 206 varied.

208 We performed the same set of analyses on both experiments. The results from both experiments were very 209 similar.

211 To be able to directly compare performance in the detection and discrimination tasks, we used accuracy (the 212 percentage of correct responses) as our dependent measure. However, the results for the detection task are 213 similar when using $d^{\prime}$ (a measure of sensitivity that is based on signal-detection theory). Mean accuracy on the 214 detection task was $74.7 \%$ (Exp 1) and 73.2\% (Exp 2). Mean accuracy on the discrimination task was $74.5 \%$ 215 (Exp 1) and $75.2 \%(\operatorname{Exp} 2)$.

216 To test whether pupil size (as manipulated through peripheral brightness) affects performance (see Figure 3), 217 and does so differently for the discrimination and detection tasks, we conducted a generalized linear mixed 218 effects model (GLM) with response-correct as dependent variable (binomial), brightness (low [reference], 219 medium, high), condition (detection [reference], discrimination), and the brightness $\times$ condition interaction as 220 fixed effects. We included only by-participant random intercepts, because more complex models failed to 221 converge. (However, the results do not crucially depend on the exact model structure.) All mixed-effects 222 analyses were conducted with the R package lme4 (Douglas et al., 2015), in which the above model 223 corresponds to the following formula: correct brightness * condition + 224 (1) subject_nr).

225 There was an effect of brightness (Exp 1: $Z=-8.754, p<.001$; Exp 2: $Z=-8.005, p<.001)$, indicating that for 226 the detection (reference) condition, accuracy decreased with increasing brightness. There was an effect of 227 condition $(\operatorname{Exp} 1: Z=-5.343, p<.001 ; \operatorname{Exp} 2: Z=-2.001, p=.045)$ indicating that for the low (reference) 228 brightness, accuracy was lower for the discrimination than detection condition. Crucially, there was also a 229 brightness $\times$ condition interaction $(\operatorname{Exp} 1: Z=6.586, p<.001$; $\operatorname{Exp} 2: Z=4.114, p<.001)$, indicating that the 230 effect of brightness was driven by the detection condition, and not present in the discrimination condition. 
231 To confirm this, we also analyzed the discrimination dondition separately, in a model with only brightness as

232 fixed effect. Here we found no effect of brightness in $\operatorname{Exp} 1(Z=0.503, p=.615)$, and only a weak effect of 233 brightness in $\operatorname{Exp} 2(Z=-2.153, p=0.031)$.

234 Pupil size

235 The EyeLink provides pupil size in arbitrary units. To convert these units to millimeters of diameter, we first 236 recorded artificial pupils (black circles printed on white paper) of different sizes, and then determined a 237 function to convert arbitrary EyeLink pupil units (au) to pupil diameter (mm): $\mathrm{mm}=-0.0324+0.1075$ $238 \times \mathrm{au}^{0.5}$

239 Mean pupil size during the detection task was $4.4 \mathrm{~mm}(\operatorname{Exp} 1)$ and $5.1 \mathrm{~mm}(\operatorname{Exp} 2)$. Mean pupil size on the 240 discrimination task was $4.3 \mathrm{~mm}(\operatorname{Exp} 1)$ and $5.0 \mathrm{~mm}(\operatorname{Exp} 2)$.

241 Our brightness manipulation should have a large effect on pupil size. It is also possible that the task affects 242 pupil size, despite the fact that the two tasks were equally difficult. To test this, we conducted a linear mixed243 effects analysis (LMER) with pupil size (prior to the onset of the trial) as dependent measure and brightness, 244 condition, and a brightness $\times$ condition interaction as fixed effects. Again, we included only by-participant 245 random intercepts, because more complex models failed to converge (see Figure 4).

246 There was an effect of brightness (Exp 1: $t=-87.405 ; p<.001$ Exp 2: $t=-58.165, p<.001)$, reflecting that 247 pupil size decreased with increasing brightness. There was also an effect of condition (Exp 1: $t=-3.849, p<$ 248.001 ; Exp. 2: $t=-4.340, p<.001$ ), reflecting that pupil size was larger in the detection than in the 249 discrimination condition. There was no notable brightness $\times$ condition interaction $(\operatorname{Exp} 1: t=-0.930, p=$ $250 \quad 0.352 ; \operatorname{Exp} 2: t=-0.216, p=0.829)$.

\section{Discussion}

252 In summary, we found that detection performance was better with large pupils (and a dark periphery) than with 253 small pupils (and a bright periphery). This relationship was strong, robust, and in the direction that we 254 predicted. However, and unlike we predicted, we did not find that discrimination performance increased with 255 decreasing pupil size (and thus increasing peripheral brightness); in fact, there was a slight tendency in the 256 opposite direction for Exp. 2.

257 In addition, we found that pupil size was larger in the detection than in the discrimination condition, even 258 though both tasks were equally difficult. 
259 A limitation of our setup for measuring discrimination performance was that we could not present very small

260 stimuli: When presented at full contrast, even the finest possible grating (i.e. 2 px/cycle) or the smallest

261 possible letter $(5 \times 5$ pixels $)$ could be discriminated without too much trouble by someone with normal vision.

262 Therefore, to increase the difficulty of the discrimination task, we also reduced the contrast of the target 263 stimulus, and our discrimination task was therefore not a pure measure of discrimination performance (a 264 limitation that we addressed in Experiment 3).

\section{Experiment 3}

266

267

268

269

270

271

272

273

274

275

276

277

278

279

280

281

282

283

284

285

286

287

In Experiment 3, we used a setup that allowed us to present very small letters. The aim of this experiment was to investigate whether we could observe an advantage of small pupils (and increased peripheral brightness) on discrimination performance in a task that tested the limits of visual acuity. If so, this would suggest that the absence of a small-pupil benefit in Experiments 1 and 2 was due to the fact that, in these experiments, our stimuli were not sufficiently fine to test the limits of visual acuity.

\section{Methods}

Participants, Ethics, and Apparatus 20 naive observers participated in the experiment, after providing informed consent. The experiment was approved by the local ethics committee of Groningen University (16163-SP-NE and 16349-S-NE). Observers were recruited from the community of Groningen University, but for privacy reasons we did not collect specific demographic information. Pupil size was recorded with an EyeLink 1000 (SR Research). Stimuli were presented with OpenSesame 3.1 (Mathôt et al., 2012) on three separate 7" tablets (Samsung Galaxy Tab 7), each with a resolution of $1280 \times 800$ px. Two tablets were presented nearby, at angle of about $50^{\circ}$ (relative to the direction of gaze) and a distance of about $50 \mathrm{~cm}$ on both sides of the participant's head, and served as light sources (see Figure 5a). One tablet was placed in front of the participant, at a distance of $5.5 \mathrm{~m}$, and served as the target display.

Task and Design Each trial started with the presentation of a black central fixation $\operatorname{dot}\left(\mathrm{R}=0.11^{\circ}[90 \mathrm{px}]\right)$ for $500 \mathrm{~ms}$ on the target display. Next, a lowercase or uppercase letter ('a', 'b', 'd', 'e', 'g', 'h', 'l', 'm', 'n', 'q', ' $\mathrm{r}$ ', or ' $\mathrm{t}$ ') was presented for $2.5 \mathrm{~s}$. Letters were presented centrally in black monospace font (Droid Sans Mono). The size of the letters was varied with a Quest adaptive procedure to converge on $75 \%$ accuracy; this resulted in a range of vertical letter sizes between roughly $0.25^{\circ}$ and $0.08^{\circ}$ (visual degrees). Participants pressed the ' $z$ ' key to indicate that the letter was lowercase, and the ' $/$ ' key to indicate that the letter was uppercase.

Peer) reviewing PDF | (2019:05:37249:2:0:NEW 5 Nov 2019) 
288 The experiment started with a practice block of 75 trials during which the background of all tablets was gray

$289\left(72.15 \mathrm{~cd} / \mathrm{m}^{2}\right.$ for target display; $69.01,64.70 \mathrm{~cd} / \mathrm{m}^{2}$ for peripheral displays). This practice block also served to 290 determine an appropriate font size to start with during the experimental blocks. Next, participants performed 291 six experimental blocks during which the brightness of the light-source tablets was varied $\left(343.30 \mathrm{~cd} / \mathrm{m}^{2}\right.$ and $292345.20 \mathrm{~cd} / \mathrm{m}^{2}$ for the two peripheral displays], medium $\left[69.01 \mathrm{~cd} / \mathrm{m}^{2}, 64.70 \mathrm{~cd} / \mathrm{m}^{2}\right]$, or dark $\left[0.83 \mathrm{~cd} / \mathrm{m}^{2}, 0.88\right.$ $\left.293 \mathrm{~cd} / \mathrm{m}^{2}\right]$ ) while the background of the target display remained gray (see Figure $5 \mathrm{~b}$ ). Each experimental block 294 started with the font size that the practice block had ended with. The order of the experimental blocks followed 295 a counterbalanced $\mathrm{ABCABC}$ design. In total, participants performed 375 trials in approximately 40 minutes.

297 Task performance

298 For each participant and brightness level separately, we took the final Quest test value as a measure of 299 performance (font size was determined based on the Quest value, and the final Quest value corresponded to a 300 font size resulting in $75 \%$ accuracy). To test whether peripheral brightness (and pupil size) affects performance, we conducted a linear mixed effects model (LME) with final Quest value as dependent measure and brightness as fixed effect. We included by-participant random intercepts and slopes. There was an effect of brightness $(t=-2.356, p=.029)$, indicating that discrimination performance increased with increasing peripheral brightness (Figure 6).

If pupil size affects performance, then we should not only find differences between the conditions in which we experimentally manipulate pupil size, but also between participants that differ in the size of their pupils. The fact that we had increased the number of participants from 9 (in Exp 1 and 2) to 20 (in Exp 3) made it feasible to look at whether individual differences in pupil size affect performance. To do so, we determined, for each participant separately, the mean pupil size during the entire experiment, and the average final Quest value (averaged across brightness levels). There was a correlation between the two measures $(r=-.508, p=.022)$, indicating that participants with smaller overall pupils had higher performance; however, this correlation was largely driven by one participant with especially large pupils, and after excluding this participant the correlation was no longer reliable $(r=-.259, p=.284)$.

\section{Pupil size}

315 To test whether our brightness manipulation affects pupil size, we conducted an LME with pupil size as dependent measure and brightness as fixed effect. We included by-participant random intercepts and slopes.

317 There was an effect of brightness $(t=-12.662, p<.001)$, indicating that pupil size decreased with increasing 318 brightness of the flanking tablets. Pupil size was converted from arbitrary units to millimeters of diameter with the same procedure as used for Experiments 1 and 2. 
321 As predicted, we found that small pupils (and increased peripheral brightness) improved discrimination 322 performance. In, addition we found some evidence that participants with small pupils had higher 323 discrimination performance, although this result was driven largely by a single participant with very large 324 pupils. That is, there was a clear link between pupil size and discrimination performance, at least when pupil 325 size was manipulated experimentally, and possibly also when considering individual differences.

\section{General Discussion}

327 Here we report that pupil size, when manipulated through peripheral brightness, has qualitatively different 328 effects on discrimination of fine stimuli in central vision and detection of faint stimuli in peripheral vision.

329 Specifically, we found that small pupils (and thus a bright periphery) are associated with improved 330 discrimination of small letters presented in central vision. This is consistent with previous studies that showed 331 a so-called positive-polarity advantage; that is, it is easier to read dark letters on a bright background (positive 332 polarity) than it is to read bright letters on dark background (negative polarity) (Buchner et al., 2009; Dobres et 333 al., 2017; Piepenbrock et al., 2014b, 2014a; Taptagaporn \& Saito, 1990). We observed this association only 334 (but highly reliably) with very small letters that were at the limits of visual acuity. This is consistent with a 335 previous study showing that the positive-polarity advantage is most pronounced for small letters (Piepenbrock 336 et al., 2014a). The small-pupil benefit for discrimination is likely due to the fact that visual acuity is highest 337 with small pupils, which suffer less from optical distortions that reduce visual acuity (Campbell \& Gregory, 338 1960; Liang \& Williams, 1997; M. Lombardo \& Lombardo, 2010; Woodhouse, 1975; Bombeke et al., 2016).

339 We further found that large pupils (and thus a dark periphery) are associated with improved detection of faint 340 stimuli that were presented at an unpredictable location in peripheral vision. This large-pupil benefit for 341 detection is likely due to two factors. First, large pupils increase light influx, which increases the signal-to342 noise ratio of vision, which in turn facilitates detection of very faint stimuli (but perhaps not, or hardly, of 343 stimuli that are presented well above the detection threshold, as used for example by Thigpen et al., [2018]).

344 Second, the dark periphery that we used to induce large pupils resulted in reduced light scatter (M. Lombardo $345 \&$ Lombardo, 2010), in turn resulting in increased image contrast, thus making it easier to detect stimuli. 346 Therefore, reduced light scatter likely also contributed to the large-pupil benefit (which is therefore in part 347 likely a dark-periphery benefit). 
348 Our results offer a possible explanation for why pupils dilate in response to increased arousal (e.g. Mathôt,

349 2018; Mathôt \& Van der Stigchel, 2015). Situations that require fine discrimination are often characterized by

350 low levels of arousal, and situations that require detection are often characterized by high levels of arousal. For

351 example, arousal is low when a person is reading a book, or when an animal is foraging for food. In such cases,

352 it is crucially important to identify what you're looking at. In contrast, arousal is high when a person is afraid,

353 or when an animal is on the lookout for predators. In such cases, it is crucially important to detect unexpected

354 dangers. In other words, pupil dilation in response to arousal may reflect an increased emphasis on visual

355 sensitivity, at the expense of visual acuity, to meet the demands of the situation.

356 An incidental yet striking result is that pupil size was larger during the detection task than during the

357 discrimination task. Because there was no systematic difference in difficulty between the two tasks, this pupil-

358 size difference is likely not due to differences in mental effort (which is known to affect pupil size, see e.g.

359 Mathôt, 2018). One possibility is that, in the detection task, the pupil dilated as a result of attention being

360 directed to peripheral rather than central vision (cf. Brocher, Harbecke, Graf, Memmert, \& Hüttermann, 2018;

361 Daniels, Nichols, Seifert, \& Hock, 2012). An even more interesting possibility is that the pupil automatically

362 assumes a size that is optimal for the current task, and that arousal-related pupil responses are merely one

363 example of this general principle.

364 Our results also have implications for the ergonomics of display design. The idea that information is best 365 presented as dark stimuli against a bright background (positive polarity) is well-established within human366 factors research (Buchner et al., 2009; Dobres et al., 2017; Piepenbrock et al., 2014b, 2014a; Taptagaporn \& 367 Saito, 1990). Our results confirm this idea, but add the important caveat that this may only hold for displays 368 that contain highly detailed information that should be discriminated; an example of such a display would be a 369 web page that contains text in a small font. In contrast, displays that emphasize detection over discrimination 370 may function best when bright stimuli are presented against a dark background (negative polarity); an example 371 of such a display might be an air-traffic control display. More generally, whether a positive or a negative 372 polarity is best depends on many factors, some of which we have highlighted in the present study. But there 373 are many additional factors that we have not considered here, including the role of light adaptation, discomfort 374 glare, contrast sensitivity, and the extent to which central or peripheral vision is used (Kalloniatis \& Luu, 375 1995).

376 Finally, our results also speak to the recent debate about whether pupil size has been a confound in previous 377 studies in the field of cognitive neuroscience. Bombeke and colleagues (2015) have argued that pupil size 378 affects early visual processing; this implies that whenever an experimental manipulation affects pupil size, any 379 difference in brain responses may be due to differences in pupil size. In contrast, Thigpen and colleagues 380 (2018) have argued that differences in pupil size do not markedly affect early visual processing; this implies 
381 that researchers can safely interpret differences in brain responses between conditions, even if these are 382 accompanied by differences in pupil size. Our own position is somewhere in between. It seems that differences 383 in pupil size can measurably affect behavior and, since behavior originates from the brain, also brain activity. 384 However, we induced pupil-size differences that were far larger than the few-percent change that is generally 385 observed in cognitive-neuroscience experiments. And even so, we had to go through great lengths in order to 386 find a measurable effect on visual acuity. In other words, in most previous experiments the confounding effect 387 of pupil size has likely been small.

388 In summary, we have shown that small pupils, induced through a bright periphery, are associated with 389 improved discrimination of fine stimuli in central vision. In contrast, large pupils, induced through a dark 390 periphery, are associated with improved detection of faint stimuli in peripheral vision. 


\section{References}

395

396

397

398

399

400

401

402

403

404

405

406

407

408

409

410

411

412

413

414

415

416

417

418

419

420

421

422

423

424

425

Ajasse, S., Benosman, R. B., \& Lorenceau, J. (2018). Effects of pupillary responses to luminance and attention on visual spatial discrimination. Journal of Vision, 18(11), 6-6. http://doi.org/10.1167/18.11.6

Binda, P., Pereverzeva, M., \& Murray, S. O. (2013). Attention to bright surfaces enhances the pupillary light reflex. Journal of Neuroscience, 33(5), 2199-2204. http://doi.org/10.1523/jneurosci.3440-12.2013

Bombeke, K., Duthoo, W., Mueller, S. C., Hopf, J., \& Boehler, N. C. (2016). Pupil size directly modulates the feedforward response in human primary visual cortex independently of attention. NeuroImage, 127, 6773. http://doi.org/10.1016/j.neuroimage.2015.11.072

Brocher, A., Harbecke, R., Graf, T., Memmert, D., \& Hüttermann, S. (2018). Using task effort and pupil size to track covert shifts of visual attention independently of a pupillary light reflex. Behavior Research Methods. http://doi.org/10.3758/s13428-018-1033-8

Buchner, A., Mayr, S., \& Brandt, M. (2009). The advantage of positive text-background polarity is due to high display luminance. Ergonomics, 52(7), 882-886. http://doi.org/10.1080/00140130802641635

Campbell, F. W., \& Gregory, A. H. (1960). Effect of size of pupil on visual acuity. Nature, 4743, 1121-1123. http://doi.org/10.1038/1871121c0

Carandini, M., \& Heeger, D. J. (2012). Normalization as a canonical neural computation. Nature Reviews Neuroscience, 13(1), 51-62. http://doi.org/10.1038/nrn3136

Crawford, B. H. (1936). The dependence of pupil size upon external light stimulus under static and variable conditions. Proceedings of the Royal Society B: Biological Sciences, 121(823), 376-395. https://doi.org/10.1098/rspb.1936.0072

Daniels, L. B., Nichols, D. F., Seifert, M. S., \& Hock, H. S. (2012). Changes in pupil diameter entrained by cortically initiated changes in attention. Visual Neuroscience, 29(02), 131-142. http://doi.org/10.1017/s0952523812000077

Dobres, J., Chahine, N., \& Reimer, B. (2017). Effects of ambient illumination, contrast polarity, and letter size on text legibility under glance-like reading. Applied Ergonomics, 60, 68-73. http://doi.org/10.1016/j.apergo.2016.11.001

Douglas Bates, Martin Maechler, Ben Bolker, Steve Walker (2015). Fitting linear mixed-effects models using lme4. Journal of Statistical Software, 67(1), 1-48. http://doi.org/10.18637/jss.v067.i01

Kalloniatis, M., \& Luu, C. (1995). Visual acuity. In H. Kolb, E. Fernandez, \& R. Nelson (Eds.), Webvision: The Organization of the Retina and Visual System. Retrieved from https://webvision.med.utah.edu/ Keuleers, E., \& Brysbaert, M. (2010). Wuggy: A multilingual pseudoword generator. Behavior Research Methods, 42(3), 627-633. http://doi.org/10.3758/BRM.42.3.627 
426 Keuleers, E., Diependaele, K., \& Brysbaert, M. (2010). Practice Effects in Large-Scale Visual Word

427 Recognition Studies: A Lexical Decision Study on 14,000 Dutch Mono- and Disyllabic Words and

428 Nonwords. Frontiers in Psychology, 1. http://doi.org/10.3389/fpsyg.2010.00174

429 Liang, J., \& Williams, D. R. (1997). Aberrations and retinal image quality of the normal human eye. Journal of 430 the Optical Society of America A, 14(11), 2873-2883. http://doi.org/10.1364/josaa.14.002873

431 Lombardo, M., \& Lombardo, G. (2010). Wave aberration of human eyes and new descriptors of image optical

432

433 quality and visual performance. Journal of Cataract \& Refractive Surgery, 36(2), 313-331.

434 http://doi.org/10.1016/j.jcrs.2009.09.026

Mathôt, S. (2018). Pupillometry: Psychology, physiology, and function. Journal of Cognition, 1(1), 1-16.

436

437

438

439

440

441

442

443

444

445

446

447

448

449

450

451

452

453

454

455 http://doi.org/10.5334/joc.18

Mathôt, S., \& Van der Stigchel, S. (2015). New light on the mind's eye: The pupillary light response as active vision. Current Directions in Psychological Science, 24(5), 374-378. http://doi.org/10.1177/0963721415593725

Mathôt, S., Schreij, D., \& Theeuwes, J. (2012). OpenSesame: An open-source, graphical experiment builder for the social sciences. Behavior Research Methods, 44(2), 314-324. http://doi.org/10.3758/s13428-0110168-7

Mathôt, S., van der Linden, L., Grainger, J., \& Vitu, F. (2013). The pupillary response to light reflects the focus of covert visual attention. PLOS ONE, 8(10), e78168. http://doi.org/10.1371/journal.pone.0078168

Piepenbrock, C., Mayr, S., \& Buchner, A. (2014a). Positive Display Polarity Is Particularly Advantageous for Small Character Sizes: Implications for Display Design. Human Factors, 56(5), 942-951. http://doi.org/10.1177/0018720813515509

Piepenbrock, C., Mayr, S., \& Buchner, A. (2014b). Smaller pupil size and better proofreading performance with positive than with negative polarity displays. Ergonomics, $0(0), 1-8$. http://doi.org/10.1080/00140139.2014.948496

Taptagaporn, S., \& Saito, S. (1990). How display polarity and lighting conditions affect the pupil size of VDT operators. Ergonomics, 33(2), 201-208. http://doi.org/10.1080/00140139008927110

Thigpen, N. N., Bradley, M. M., \& Keil, A. (2018). Assessing the relationship between pupil diameter and visuocortical activity. Journal of Vision, 18(6), 7-7. http://doi.org/10.1167/18.6.7

Woodhouse, J. M. (1975). The effect of pupil size on grating detection at various contrast levels. Vision Research, 15(6), 645-648. http://doi.org/10.1016/0042-6989(75)90278-3

Peer] reviewing PDF | (2019:05:37249:2:0:NEW 5 Nov 2019) 


\section{Figure 1}

Luminance manipulation in Experiments 1 and 2.

The luminance of the visual periphery was varied to manipulate pupil size. All task-relevant stimuli appeared on a central gray disk that was kept constant throughout the experiment.

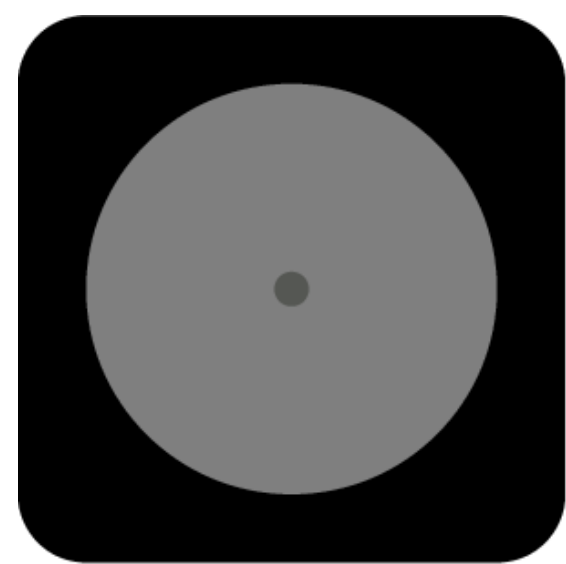

Dark (large pupil)

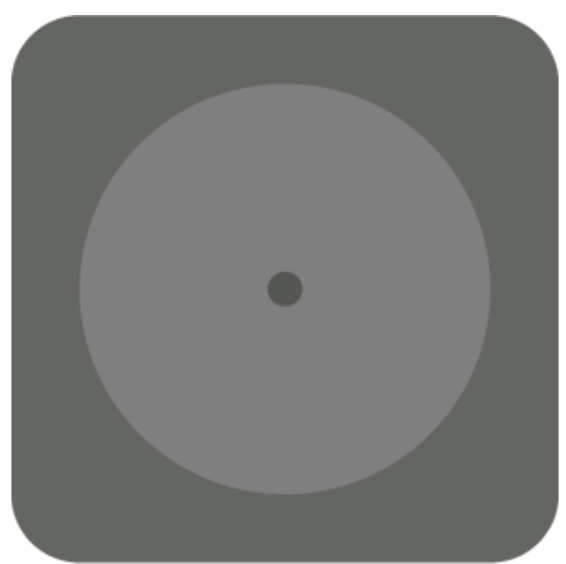

Medium (medium-size pupil) (small pupil) 
Figure 2

Schematic paradigm of Experiments 1 and 2.

a) Orientation-discrimination task for Experiment 1. b) Orientation-detection task for

Experiment 1. c) Word-discrimination (lexical decision) task for Experiment 2. d) Worddetection task for Experiment 2. 
a) Experiment 1: Orientation discrimination
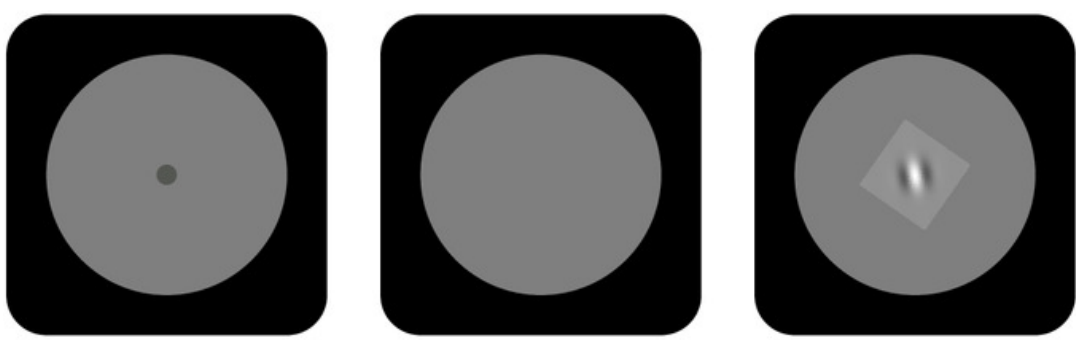

$650 \mathrm{~ms}$

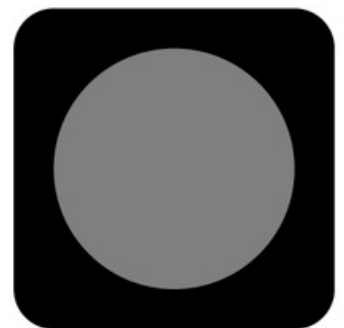

Until response or $2350 \mathrm{~ms}$

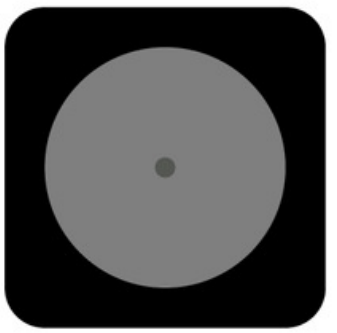
Until response or $2350 \mathrm{~ms}$

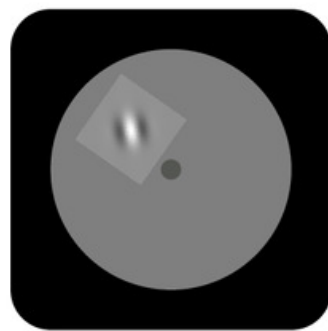

$650 \mathrm{~ms}$

(50\% of trials)

c) Experiment 2: Word discrimination
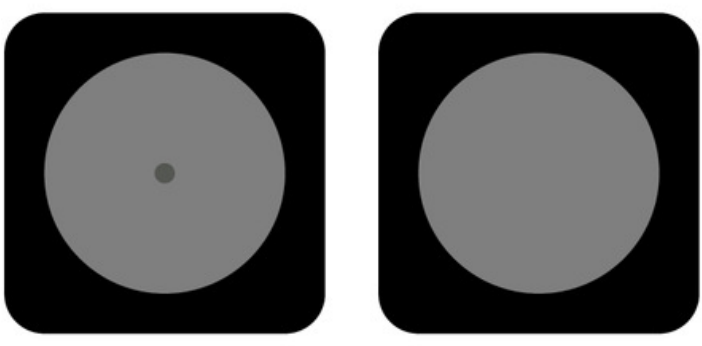

$500 \mathrm{~ms}$
$500-1500 \mathrm{~ms}$

d) Experiment 2: Word detection
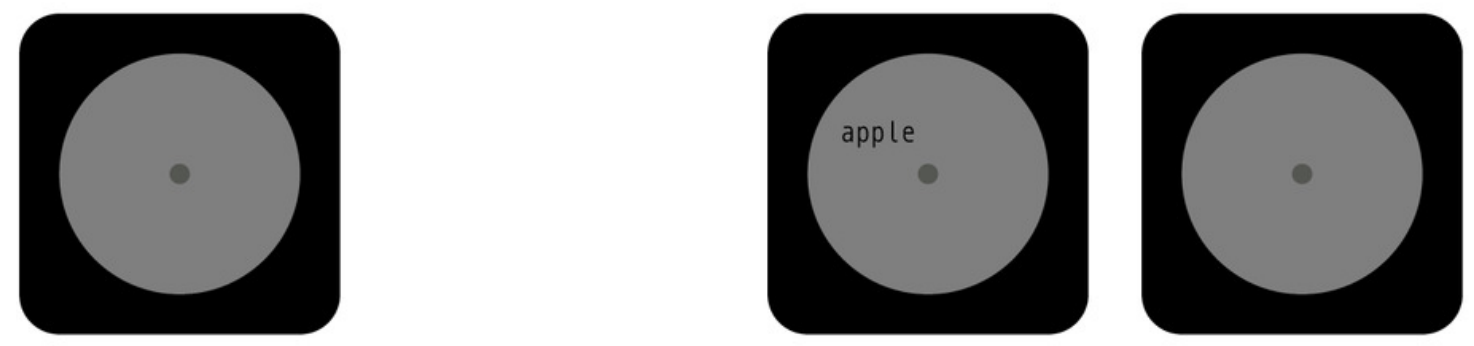

$650 \mathrm{~ms}$

(50\% of trials)
Until response

or $2350 \mathrm{~ms}$
Target present

or absent?

Target present or absent?

Word or non-word?

or $2350 \mathrm{~ms}$ 


\section{Figure 3}

Task performance as a function of pupil size in Experiments 1 and 2.

Detection accuracy increased with decreasing peripheral brightness, and thus increasing

pupil size ( $b, d$; pink lines). However, there was no effect of peripheral brightness on discrimination performance ( $a$, $c$; green lines). Gray dotted lines indicate individual participants. Colored solid lines indicate grand averages. 
a) Exp 1: Discrimination

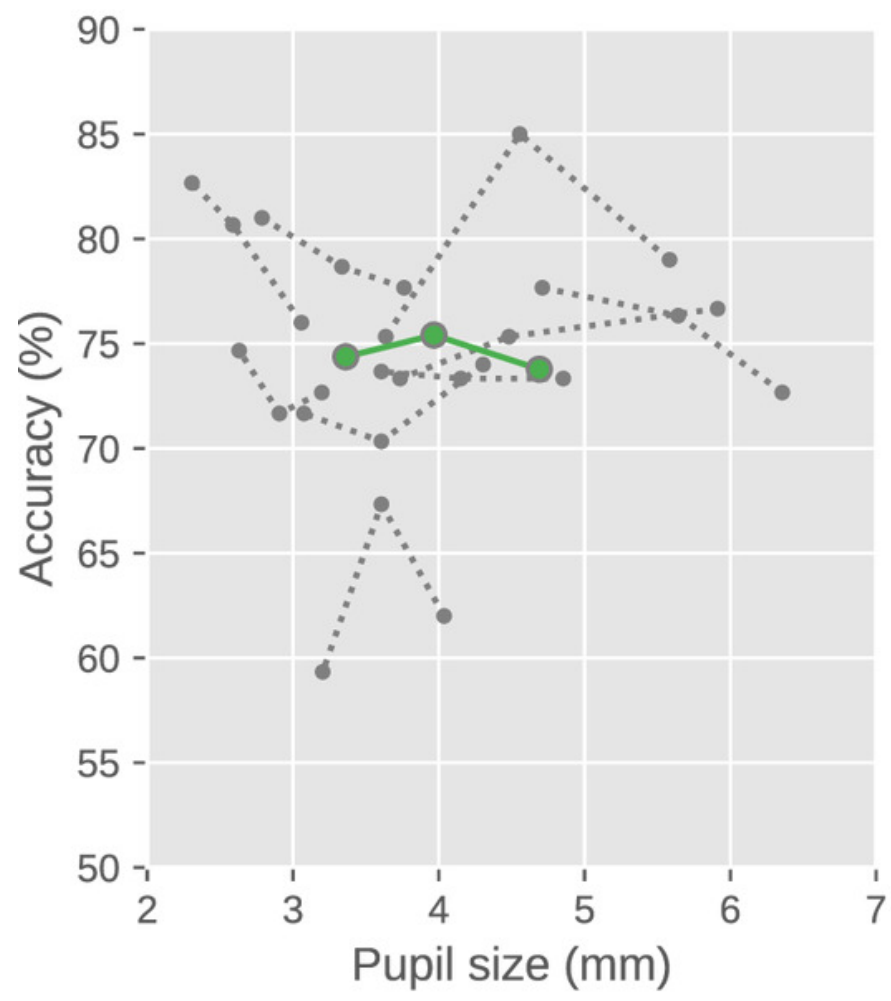

c) Exp 2: Discrimination

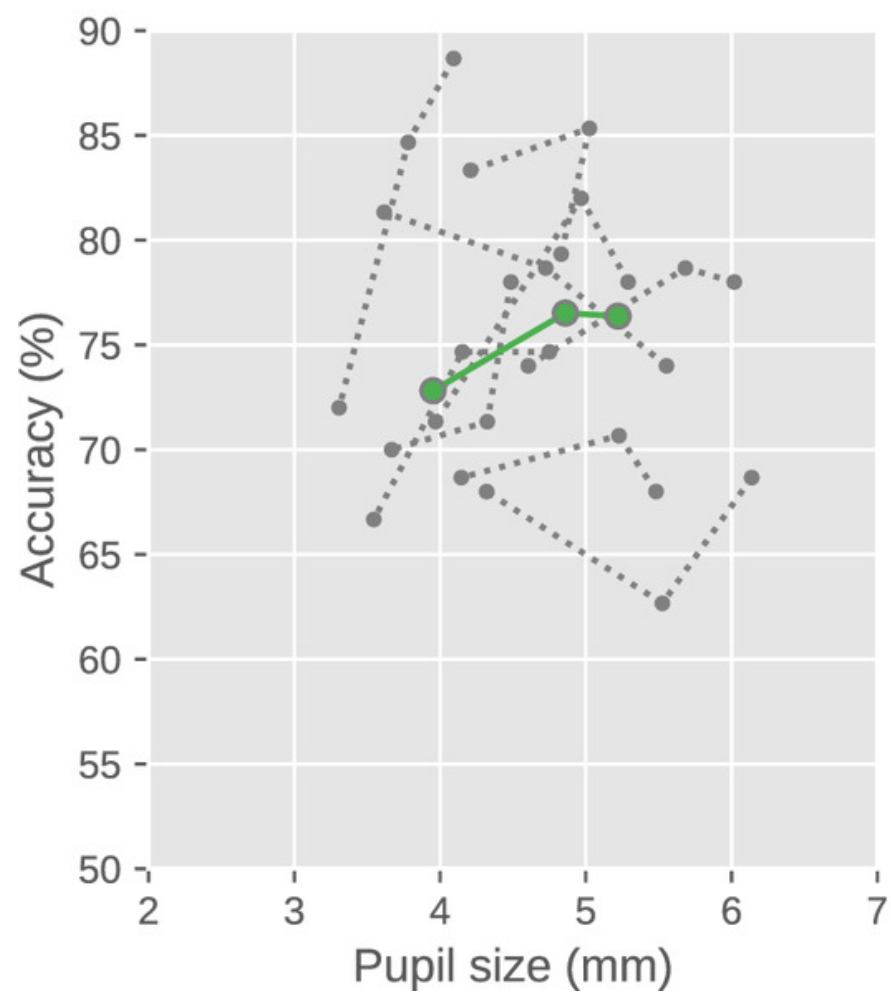
b) Exp 1: Detection
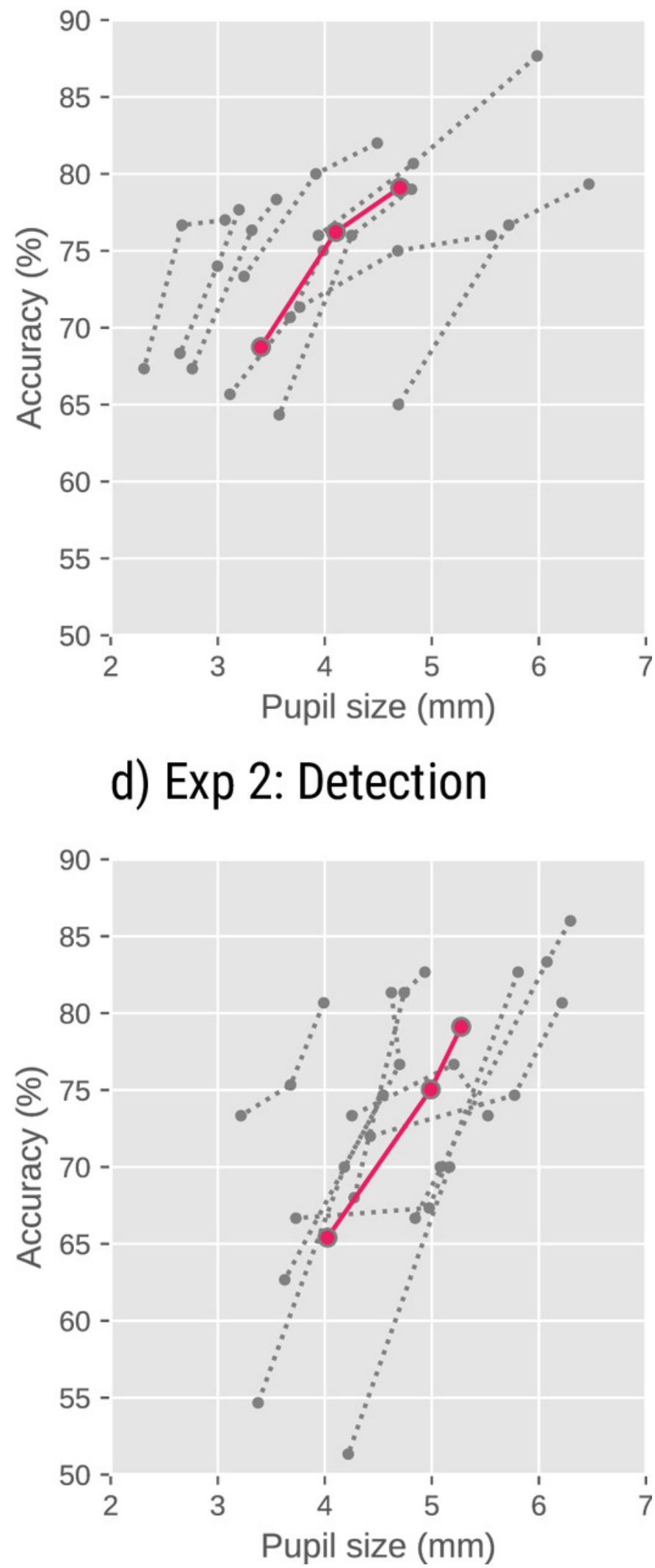
Figure 4

Pupil size as a function of task and peripheral brightness in Experiments 1 and 2.

In both experiments, pupil size decreased with decreasing peripheral brightness. In addition, pupil size was slightly larger in the Detection (pink dots) than in the Discrimination (green dots) condition.

a) Experiment 1

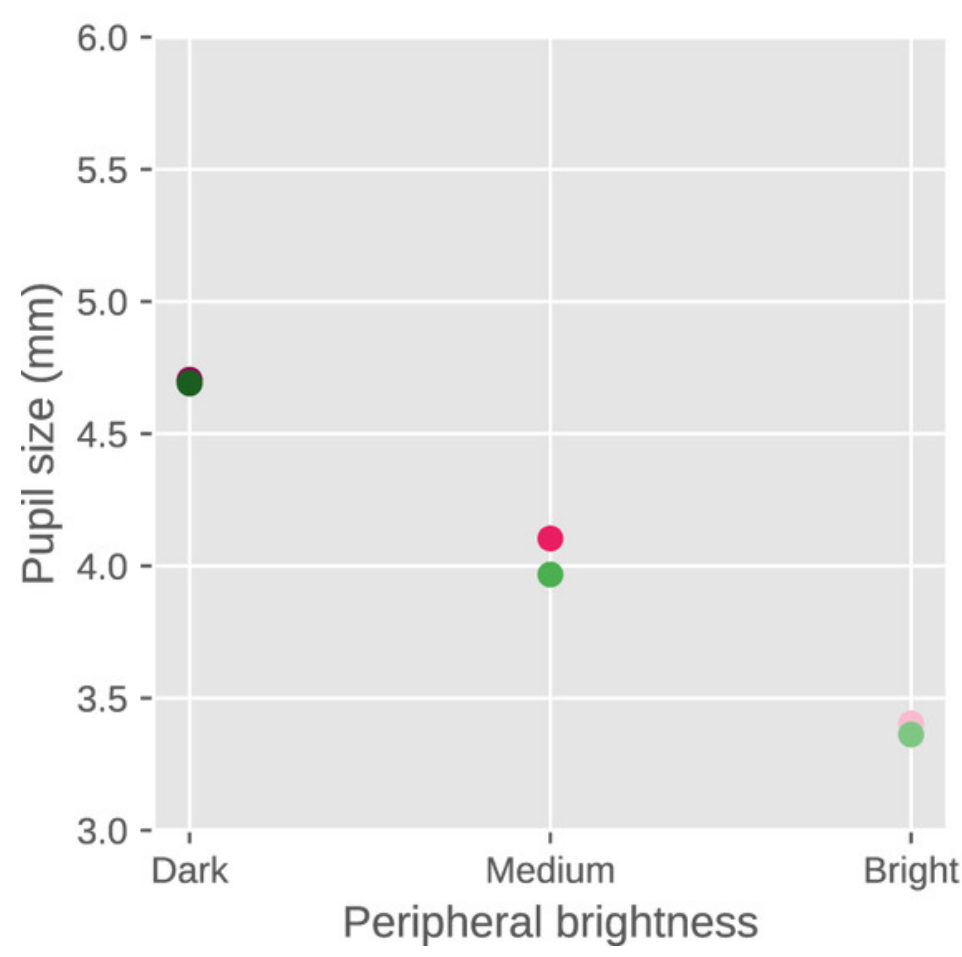

b) Experiment 2

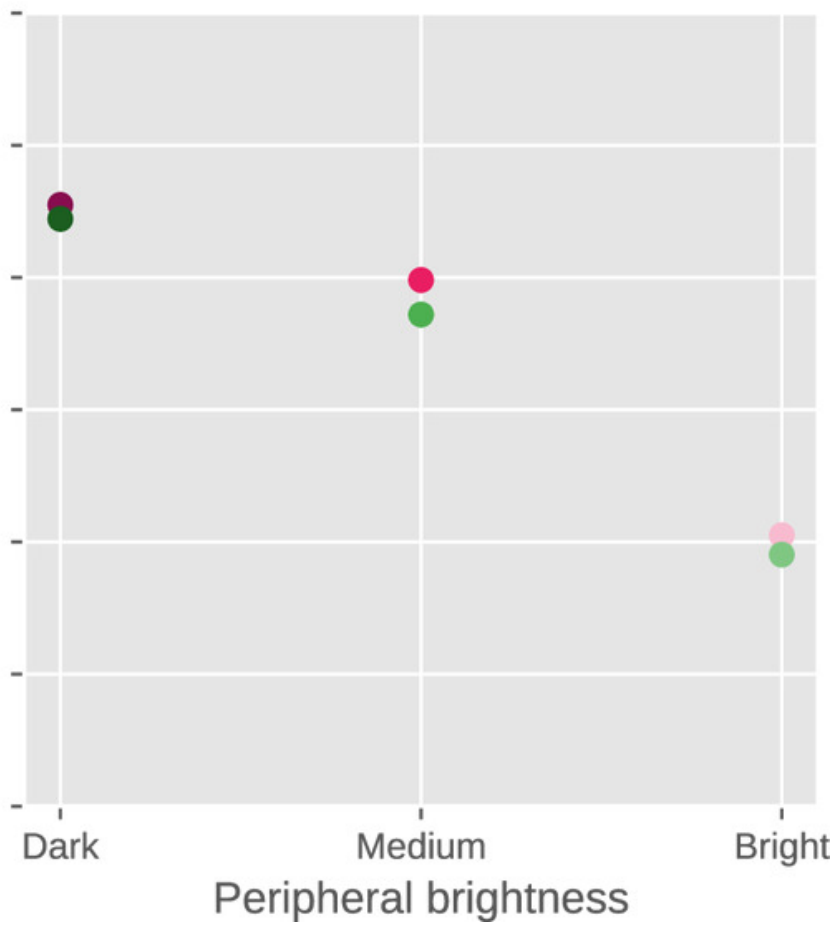




\section{Figure 5}

Schematic set-up and paradigm of Experiment 3.

a) Participants indicated whether a target letter was uppercase or lowercase. b) Pupil size was manipulated by varying the brightness of two displays that were positioned near the participant, and flanked the target display.

\section{a) Experimental set-up}

\section{b) Brightness manipulation}
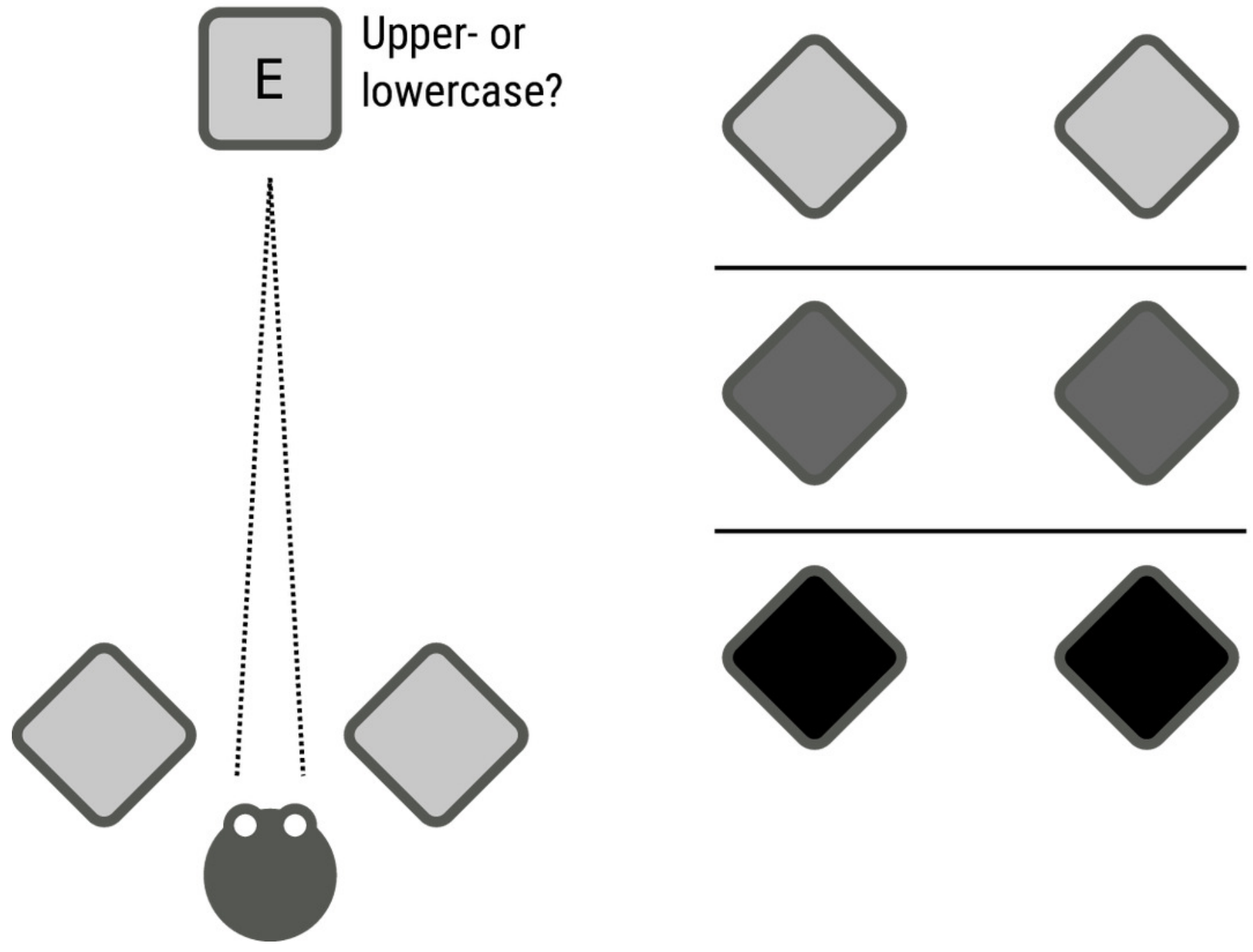


\section{Figure 6}

Visual acuity as a function of pupil size in Experiment 3.

Discrimination performance increased with increasing peripheral brightness (and decreasing pupil size). Lines correspond to individual participants (for clarity, different participants have different colors). Dots correspond to different levels of peripheral brightness, such that the highest peripheral brightness corresponds to the smallest pupil size. In addition, participants with smaller overall pupils had higher discrimination performance (although this effect was largely driven by one participant with very large pupils). The dashed line indicates the regression line. 


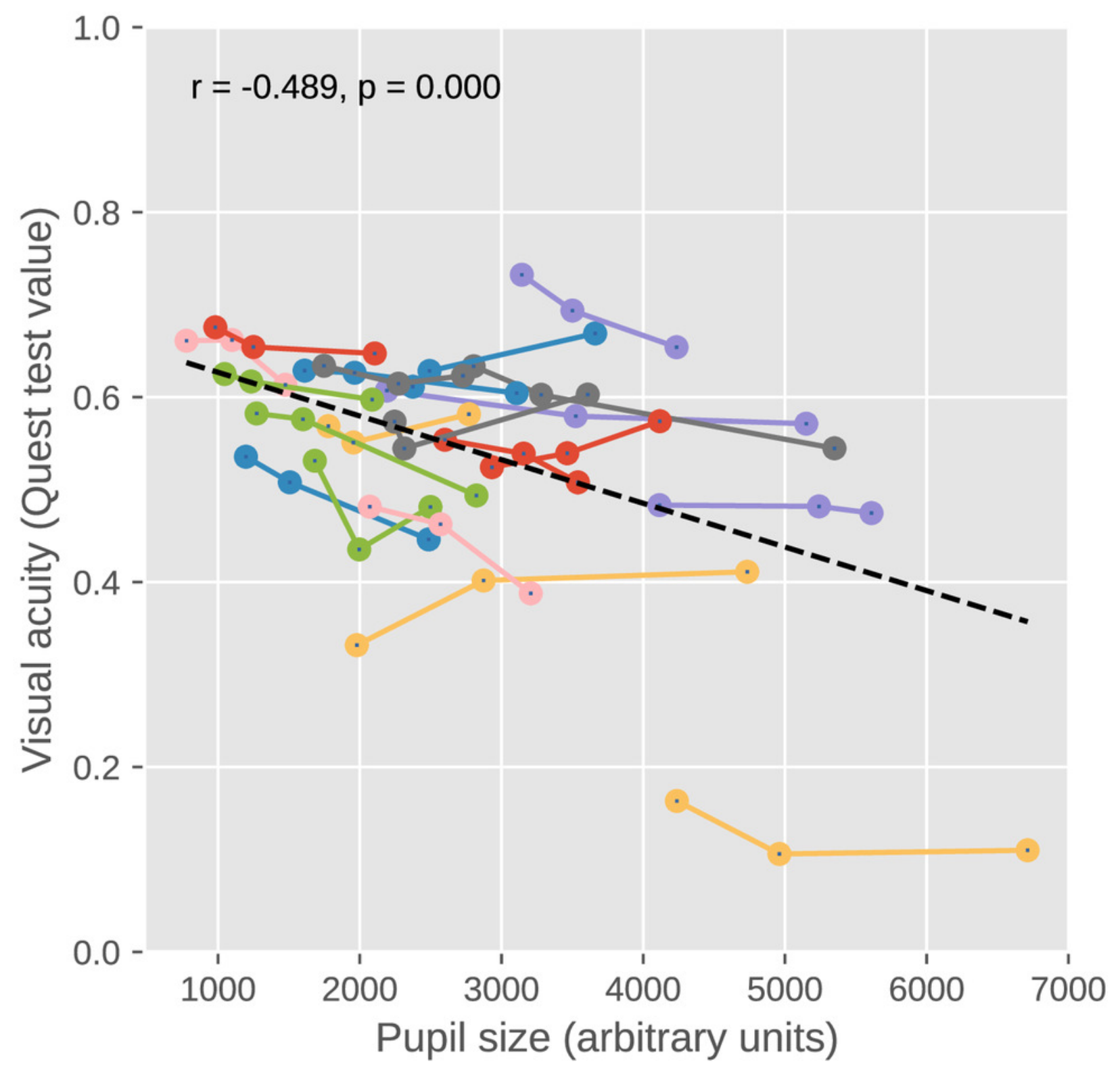

